\title{
Potentially avoidable readmissions: Understanding drivers and technology-enabled solutions
}

\author{
Aidan L $\underline{\operatorname{Tan}}{ }^{1} M P H$, Woan Shin $\underline{\operatorname{Tan}}{ }^{1}{ }{ } D$
}

Hospital admissions place high resource demands on the health system, and is a major cost-driver in Singapore and globally. ${ }^{1-3}$ Admissions have and will continue to increase given Singapore's ageing population and growing chronic disease and multimorbidity burden, impacting care quality and patient/provider experience., ${ }^{2,4}$ While majority of admissions are clinically appropriate and unavoidable as part of care provision, ${ }^{1,2,4}$ a significant proportion are readmissions for conditions arising from potentially avoidable issues occurring during the initial admission, discharge or post-admission..$^{1,3,5}$ Recognising this, Singapore included 30-day readmission rates in its performance measure and quality improvement framework for acute hospitals in 2011. ${ }^{1}$ However, healthcare expenditure continued to increase, doubling from SGD4 billion in 2011 to SGD10 billion in 2018, and was projected to triple by 2020 (SGD12 billion). ${ }^{2}$ Hence, research into effective reduction in high-cost drivers (30-day readmissions) remains imperative to ensure long-term sustainability.

The concept of potentially avoidable readmissions (PARs) is readily understood and widely applied. ${ }^{1,3}$ However, identification is difficult, requiring determination of clinical appropriateness within a given system structure and resources, subject to the greater environment and culture it is nested in., ${ }^{3,6}$ Combined with the massive volume of admissions annually, manual identification is impossible.

Administrative database algorithms from international or Singapore literature allow automation of this process. ${ }^{3,6}$ These algorithms allow a more objective identification of PARs and their prevalence, assuming such a readmission could be identified with certainty. Two approaches exist: including cases as avoidable if they match predefined a priori criteria, e.g. readmissions with the same diagnosis, and excluding admissions predefined as unavoidable, e.g. trauma, neonatal or obstetric care. ${ }^{3,6}$ The former is highly specific and identifies a much smaller number of cases but with definite preventability, while the latter is highly sensitive and identifies a large number of cases but with varying preventability. Given that preventability greatly depends upon context, the latter approach is suitable for primary screening, with further refinement of results based on local factors. One such algorithm is SQLape, upon which various internationally validated predictive scoring systems (LACE, HOSPITAL) were derived, allowing identification of patients for intervention before they incur high costs. ${ }^{3,6}$

However, such scores have demonstrated varying predictive accuracy, likely due to the overly simplified model variables. Out of practical necessity, many predictive scores are derived from administrative databases of routinely collected clinical and socio-demographic data; such data are often not comprehensive enough to adequately capture the full complexity of drivers leading to PARs. ${ }^{2,6}$ Omission of social and environmental factors may impact identification accuracy and predictive ability. ${ }^{6}$ Additionally, such factors are often specific to the Singapore context, reflecting the social, cultural and legal structures, which necessitate in-depth contextual research to uncover. ${ }^{2}$

PARs reflect a confluence of contextual issues at multiple levels: patient/individual, care provider, health system structure, and larger sociocultural environment. ${ }^{2}$ Both qualitative and quantitative methodologies are therefore essential to identify and measure the impact of these factors.

The findings by Mukhopadhyay et al. in this issue of the Annals reflect the growing recognition of socio-psychoeconomic influences upon health and clinical outcomes. ${ }^{7}$ In their mixed method study, aside from quantitative characteristics available from routine data, they attempted to uncover the qualitative differences in care journey experiences between patients with and without readmissions within 30 days. Patients with readmissions expressed more negative psychological reactions and perceptions: a more fatalistic outlook; neglect and uninvolved in care decisions; low trust in treatments; and lesser health literacy.

${ }^{1}$ Health Services and Outcomes Research, National Healthcare Group, Singapore

Correspondence: Dr Aidan L Tan, Health Services and Outcomes Research, National Healthcare Group, 3 Fusionopolis Link, \#03-08, Nexus@one-north, Singapore 138543 .

Email: aitan@nhg.com.sg 
These echo the growing body of literature, where patient psychology and perceptions and their community/ home environments influence observed health outcomes. ${ }^{2}$ These findings help build a body of evidence towards a more complete understanding of PAR drivers, and thereby increase our confidence in identification and prediction of such PARs.

Evidence suggests that reduction of PARs is possible through improvements in care quality, discharge processes and care transitions between providers in a patient-centric manner. However, it is hubris to state that all such PARs are preventable. Just as PARs are driven by a complexity of factors, interventions to prevent them would be similarly or even more so. Not all drivers are modifiable at a cost proportionate to the readmission avoided; others may require intervention at a systems or whole-society level. ${ }^{2,8,9}$

In Singapore, interventions aimed at preventing PARs are currently focused on improving care transitions via integration between providers, thereby allowing smoother discharges between care sectors and reducing the number of patients who fail to transit between providers. One such intervention is the Hospital-toHome $(\mathrm{H} 2 \mathrm{H})$ programme launched in April 2017. ${ }^{9}$

The $\mathrm{H} 2 \mathrm{H}$ programme supports care transition between hospital and health service providers through the aid of a "patient-navigator" nurse and multidisciplinary care team. Patients are identified as high-risk based on algorithm scores during their initial hospital admission. These highrisk patients are then evaluated by the $\mathrm{H} 2 \mathrm{H}$ team prior to discharge. Based on the various care needs identified and the caregivers' capability, a comprehensive care plan is made, drawing upon community care resources such as home nursing or social services as necessary.

With a reported 8,000 patients supported within a year of its roll-out, the $\mathrm{H} 2 \mathrm{H}$ programme has seen mixed effects with some patient groups showing little or even negative outcomes. ${ }^{89}$ Such programmes are primarily targeted towards a patient's medical/nursing needs and smoothening transitions between clinical providers, but often fails to address other modifiable drivers arising at the patient-level (perceptions, psychology), socioeconomic environment (income, housing), physical environment (built resources, mobility access) and the greater society (discrimination, social norms, legal and ethical structures) ${ }^{8,9}$

These results indicate the necessity of understanding the wide span of needs across different patient groups, which may extend beyond just the clinical condition. ${ }^{2,8,9}$ Transforming the patient's role from a passive recipient into an active participant in their care may further aid this process. ${ }^{10}$
Another issue lies in how technology is used (or not used) as an enabler. Current scoring systems are geared solely towards identification of patients-at-risk, as in the $\mathrm{H} 2 \mathrm{H}$ programme. This is an important first step, but doing so merely identifies the individual and fails to pinpoint the underlying reasons or domains driving this risk. ${ }^{2,3,6}$ Prediction alone is insufficient. This early truncation of digitalisation as an enabler may partially explain the lackluster results observed. The $\mathrm{H} 2 \mathrm{H}$ programme relies on assessors and human judgement to determine care needs. Without addressing the underlying drivers, intervention efforts would be misdirected and fail to evidence effectiveness. This therefore places a huge burden on the assessing team to be as comprehensive as possible, a particularly difficult task given the widespanning complexity of patient needs and perceptions across more than just the clinical conditions.

These programmes are also highly resource intensive, given the high provider-to-recipient ratio and number of services such recipients require for their complex care needs. ${ }^{8}$ It is therefore necessary to ensure that intervention efforts are appropriately directed, maximising savings from PARs avoided while minimising intervention costs. Thus, beyond leveraging technology as a prediction tool, it should be used as an enabler for interventions. Accurate and up-to-date near real-time scoring metrics should be paired with evidence-based bundled interventions across the entire patient journey. ${ }^{5,6}$

An example of how technology as an enabler could be fully utilised was implemented in a regional acute hospital. ${ }^{5}$ Using a Singapore-derived readmission risk score, the score and its risk components were embedded within the hospital's electronic medical records on a real-time basis. This allowed flagging of high-risk patients to care providers, identification of modifiable components driving the risk, and initiation of evidencebased bundled interventions specific for each riskstrata; thereby most effectively and efficiently reducing readmission risk. ${ }^{5}$

In recent years, the benefits of electronic medical records and big data are increasingly evident as supports towards an intelligent and integrated care system. They can be a powerful tool towards reducing PARs if appropriately applied in a problem-focused manner across the entire patient care journey. In the above hospital, the technology was employed as an enabler towards improving patient care: at the start of admission (risk prediction), during the admission (areas for intervention) and discharge (reduction in risk). ${ }^{5}$ Future work should include the patients' and caregivers' perspectives, either from clinical team inputs or via artificial intelligence algorithms in big data, to 
encompass risk drivers for improved prediction and solution generation.

Reducing PARs is a potential area for decreasing health system demand and cost. However, current scoring methods for identification and prediction of PAR risk is limited. Recent research shows the great complexity and interplay between socio-psychoeconomic influences driving this risk, elicitable from patient/caregiver perspectives. Engaging patients and caregivers as active partners in care is essential. Beyond simply flagging patients as high-risk based on prediction, digitalisation can and should be utilised as an enabler for interventions, informed by and targeted towards these drivers across the care journey.

\section{REFERENCES}

1. Lim E, Matthew N, Mok W, et al. Using hospital readmission rates to track the quality of care in public hospitals in Singapore. BMC Health Serv Res 2011;11(Suppl 1):A16.

2. Low LL, Tay WY, Ng MJM, et al. Frequent hospital admissions in Singapore: Clinical risk factors and impact of socioeconomic status. Singapore Med J 2018;59:39-43.
3. Yam $\mathrm{CH}$, Wong EL, Chan FW, et al. Measuring and preventing potentially avoidable hospital readmissions: A review of the literature. Hong Kong Med J 2010;16:383-9.

4. Low LL, Liu N, Lee KH, et al. FAM-FACE-SG: A score for risk stratification of frequent hospital admitters. BMC Med Inform Decis Mak 2017; 17:35.

5. Wu CX, Suresh E, Phng FWL, et al. Effect of a real-time risk score on 30-day readmission reduction in Singapore. Appl Clin Inform 2021;12:372-82.

6. Zhou H, Della PR, Roberts P, et al. Utility of models to predict 28-day or 30-day unplanned hospital readmissions: An updated systematic review. BMJ Open 2016;6:e11060.

7. Mukhopadhyay A, Mohankumar B, Chong LS, et al. Factors and experiences associated with unscheduled 30-day hospital readmission: A mixed method study. Ann Acad Med Singap 2021:50:751-64

8. Ang YH, Ginting ML, Wong CH, et al. From hospital to home: Impact of transitional care on cost, hospitalisation and mortality. Ann Acad Med Singap 2019;48:333-7.

9. Ng SCW, Kwan YH, Yan S, et al. The heterogeneous health state profiles of high-risk healthcare utilizers and their longitudinal hospital readmission and mortality patterns. BMC Health Serv Res 2019;19:931.

10. Senot C, Chandrasekaran A, Ward P, et al. The impact of combining conformance and experiential quality on hospitals' readmissions and cost performance. Management Science 2015;62:829-48.

11. Low LL, Vasanwala FF, Ng LB, et al. Effectiveness of a transitional home care program in reducing acute hospital utilization: A quasi-experimental study. BMC Health Serv Res 2015;15:100. 